\title{
Fernand Nouvion, génie de la traction électrique
}

Jean-François Picard

\section{OpenEdition}

\section{Journals}

Édition électronique

URL : https://journals.openedition.org/rhcf/2001

DOI : 10.4000/rhcf.2001

Éditeur

Rails \& histoire

Édition imprimée

Date de publication : 1 février 2003

Pagination : 229-249

ISBN : 0996-9403

ISSN : 0996-9403

Référence électronique

Jean-François Picard, «Fernand Nouvion, génie de la traction électrique », Revue d'histoire des chemins de fer [En ligne], 26 | 2003, mis en ligne le 22 janvier 2015, consulté le 22 avril 2022. URL : http:// journals.openedition.org/rhcf/2001; DOI : https://doi.org/10.4000/rhcf.2001 


\title{
Fernand Nouvion, génie de la traction électrique
}

\author{
Génie : esprit (ou démon) domestique qui régit les destinées de la maison \\ (Le Robert)
}

De Marc Seguin, l'un des inventeurs de la locomotive à vapeur, à André Chapelon qui l'a menée à son apogée un siècle plus tard, on sait le rôle majeur tenu par les ingénieurs français dans l'histoire des chemins de fer. André Chapelon, Genius of French Steam est le titre de la première biographie ${ }^{1}$ de l'illustre vaporiste qui, s'il n'avait inventé la locomotive à vapeur, en avait poussé les perfectionnements à un degré tel que certains déploraient de la voir remplacée un jour par sa concurrente électrique. Les historiens anglo-saxons qui sont plus familiers de l'histoire des techniques que leurs collègues de ce côté de la Manche confirment ainsi la renommée internationale de nos ingénieurs. Indiscutablement, Fernand Nouvion (décédé en 1998) fut l'un de ceux-ci. Ce qui ne constitue pas son seul point commun avec son confrère Chapelon. En effet, comme lui ingénieur à la SNCF, s'il n'a pas plus inventé la locomotive électrique que son alter ego la locomotive à vapeur, il n'a pas moins contribué à porter la première au degré de perfection technique qu'on lui connait aujourd'hui².

Fernand Nouvion est né le 2 novembre 1905 à Neuilly-sur-Seine dans une famille de la bourgeoisie aisée. Son père était fonctionnaire et le jeune Fernand fit ses études secondaires dans l'établissement chic de la banlieue parisienne qu'est le lycée Pasteur. Sa vocation cheminote serait née d'un superbe cadeau offert pour Noël 1912, l'un des premiers

1- Rogers, H. C. B., André Chapelon, Genius of French Steam, Londres, Ian Allan, 1972 (trad. française par B. Escudié et J. Payen, André Chapelon (1892-1978), Paris, CNRS Éditions, 1992)

2- Cet article a été rédigé grâce au concours de l'Association pour l'histoire des chemins de fer et à l'efficacité de sa secrétaire scientifique, M.-N. Polino. Il convient de mentionner aussi des entretiens réalisés par l'auteur de ces lignes avec MM.

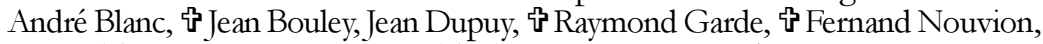
Marcel Tessier et Yves Machefert-Tassin qui lui ont apporté en diverses occasions leurs témoignages, ainsi que la mise à la disposition de l'auteur, par son gendre M. André Blanc, des notes journalières de M. Garreau (deux cahiers, couvrant la période 13 août 1940 au 6 octobre 1955). 
trains électriques Märklin. Lors de ses études à l'École supérieure d'électricité (ESE), certains de ses condisciples se souviennent d'une passion pour le chemin de fer qui ne manquait pas de le faire passer pour un original dans une école chargée de former les servants de la nouvelle industrie. Mais il en gardera un assez bon souvenir pour y revenir plus tard en tant que professeur de traction électrique (de 1957 à 1971) ${ }^{3}$. Son diplôme en poche et après un bref passage dans l'industrie, il cherche une place au chemin de fer, d'abord dans la plus « électrique » des grandes compagnies de l'époque, le Paris-Orléans (P.O.), dont Hippolyte Parodi avait entrepris d'électrifier les grandes lignes. Non sans humour, il rappelait qu'il y fut accueilli pour s'entendre conseiller de s'adresser plutôt aux Chemins de fer de l'État (Ouest), « ...où on était moins regardant sur la qualité des candidats » (!). C'est ainsi qu'il est recruté au réseau de l'État, en 1930, alors dirigé par Raoul Dautry et sur le point de lancer l'électrification de la ligne Paris-Le Mans. Affecté au service du Matériel, Nouvion évoquait les débuts de sa carrière cheminote en usant de l'humour un tantinet provocateur qui le caractérise.

«Peu après mon entrée au chemin de fer, j’ai compris que j'étais entré dans un service dévalué ! Celui du Matériel et Traction. Un jour j’ai entendu Dautry déclarer que pour la Voie et le Matériel on pouvait prendre n'importe qui. C'est facile, cela marche toujours. En ce qui concerne l'Exploitation, c'est une autre affaire. Il faut non seulement des hommes intelligents, remarquablement instruits, mais qu'ils aient en plus un don : celui de prévoir ce qui va se passer. Et Dautry ajoutait, peut-être pour nous consoler, "je n’en ai jamais rencontré un seul"... ${ }^{4} »$

Mais Nouvion reconnaissait à son premier directeur les qualités d'un grand exploitant. Il rappelle qu'on lui devait la modernisation de la banlieue Montparnasse avec ses quais hauts et un nouveau matériel de banlieue révolutionnaire pour l'époque, les rames Budd en acier inoxydable, même si le jeune ingénieur ne manquait pas de railler leurs marchepieds rabattables et leur « épouvantable quincaillerie de relais électro-pneumatiques dont le quart était toujours en panne ». Sous la férule de Marcel Garreau (X-Télécoms), le responsable de l'électrification

3- Les cours de Nouvion à SupElec ont été publiés : Électrification ferroviaire, Paris, Ecole supérieure d'électricité, 1969.

4- F. Nouvion, première « grande conférence » donnée à la SNCF (s.d.)., Dossier Nouvion (F. Nouvion avait remis un exemplaire des ses articles publiés àl'AHICF. Le reste de ses éventuels papiers techniques et de sa documentation n'a pas été retrouvé après son décès). 
Paris-Le Mans, il a participé à la mise au point de rames automotrices de ramassage, des engins remarquables destinés à assurer une desserte omnibus rapide, tout en déplorant - déjà ! - que l'Exploitation ne s'intéresse pas vraiment à la vitesse. A la création de la SNCF, en 1938, lorsque Dautry quitte le chemin de fer il propose à Nouvion de l'emmener avec lui dans la construction aéronautique, ce à quoi l'intéressé aurait répondu qu'ayant tellement appris à aimer le chemin de fer sous son autorité, il préférait y rester.

En août 1940, au lendemain de "l'étrange défaite ", Fernand Nouvion devient donc chef d'arrondissement Matériel des Batignolles (SNCF Région Ouest), chargé des ateliers d'entretien de La Folie à Nanterre. Là, il va donner la preuve de ses remarquables talents d'organisateur, non sans rencontrer quelques problèmes liés aux circonstances de cette époque tragique. Les ateliers de la Folie sont responsables de la révision générale (R.G.) des locomotives de la ligne Paris-Le Mans, une opération qui consiste à démonter complètement la machine pour vérifier et remplacer ses organes usés. Elle ne nécessitait jusque-là pas moins d'une quinzaine de jours en atelier. Il introduit aux ateliers de la Folie de nouvelles méthodes de travail, imaginant une planification raisonnée des tâches d'entretien (grosses opérations à mener en priorité), ce qui permet d'augmenter le nombre d'équipes susceptibles de travailler simultanément sur une locomotive. Il met en place un système de contrôle de qualité (essai des sous-ensembles de la locomotive, freins, contacteurs, etc.) avant leur remontage définitif ce qui réduit la période d'essais lors de la remise en service, etc. Ainsi, il obtient une réduction à cinq jours du temps nécessaire à la révision générale des locomotives 2D2 État. Ce remarquable résultat l'amène à publier en 1942 son premier article dans la Revue générale des chemins de fer sous le titre éloquent de "réduction des immobilisations du matériel $»^{5}$. Évidemment, à l'époque l'occupant allemand étant le principal client de la SNCF, cette diminution des indisponibilités lui bénéficiait au premier chef et son zèle semble avoir valu à Nouvion quelques désagréments. A la Libération, il passe devant un comité d'épuration et, si on ne peut lui reprocher des faits avérés de collaboration, son comportement ne manque pas d'évoquer quelque syndrome de type «pont de la rivière Kwaï »; on connait le film de David Lean qui raconte l'histoire (vraie) d'un officier du génie britannique fait prisonnier par les Japonais en Birmanie mais qui, pris au jeu du métier, construit un pont de chemin de fer utilisé par l'ennemi. Si, contrairement à certains de ses collègues

5- F. Nouvion, «Réduction des immobilisations du matériel », Revue générale des chemins de fer, avril 1942. 
l'affaire n'eut pour lui aucune suite fâcheuse, il n'aimait pas en évoquer le souvenir'.

En 1946, Fernand Nouvion entre à la Division d'études de la traction électrique (DETE), un département créé en 1939 au sein de la SNCF pour l'électrification de son réseau ferré. Là, il seconde Marcel Garreau le remarquable électricien chargé d'électrifier la banlieue ParisMontparnasse et la ligne Paris-Le Mans avant-guerre ${ }^{7}$. La personnalité des deux hommes était profondément différente. L’un réservé et méthodique, l'autre extraverti et inventif, mais de l'avis général ils formaient le " remarquable binôme » qui allait réaliser les électrifications de la $\mathrm{SNCF}^{8}$. La DETE va donc jouer un rôle central dans une histoire que l'historien Clive Lamming découpe en deux grands chapitres : une « ère Garreau ", celle du magistère des électriciens marquée par l'adaptation du courant alternatif à fréquence industrielle en traction ferroviaire et une « ère Nouvion » caractérisée par des progrès mécaniques destinés à faire de la locomotive électrique un moyen de traction universel ${ }^{9}$. Ceux qui ont travaillé avec lui s'accordent à reconnaitre à Nouvion les capacités d'un meneur d'hommes. Il savait insuffler un esprit de « commando » à ceux qu'on ne tardera pas à appeler la "bande à Nouvion », Marcel Tessier, André Cossié et bien d'autres, même si cela agaçait plus d'un collègue au sein d'une entreprise en principe peu soucieuse du vedettariat de ses ingénieurs.

Au lendemain de la Première Guerre mondiale, une décision gouvernementale avait décidé d'unifier le système d'électrification des chemins de fer français en courant continu à la tension de $1,5 \mathrm{kV}$, le système préconisé par Hippolyte Parodi pour les lignes de la Compagnie du P.-O. (Paris-Toulouse, Paris-Bordeaux-Irun, Irun-Toulouse-Béziers, etc.). Le continu basse tension présentait un certain nombre d'avantages. Par exemple, le fait d'être délivré à demi-tension aux moteurs de traction

6- Au risque d'aller à contre courant d'une certaine historiographie, on se gardera d'instruire ici le procès de la SNCF en collaboration avec l'ennemi. Les chemins de fer français étaient pendant la guerre sous l'étroite tutelle de la puissance occupante et reprocher à la société nationale d'avoir exploité son réseau, réparé ses installations semble relever davantage de polémiques partisanes que du souci de la recherche historique.

7- André Blanc (son gendre) a conservé deux cahiers sur trois des notes professionnelles journalières de Marcel Garreau, une source de première main pour l'histoire de la DETE (voir note 2 p. 229).

8- Témoignage de Marcel Tessier.

9- Clive Lamming, Cinquante ans de traction à la SNCF : enjeux politiques et réponses techniques, Paris, CNRS Éditions, 1997. 
des locomotives permettait un fort couple de démarrage, tandis que les moteurs continus à collecteur disposent de fortes capacités de surcharge. En revanche, il présentait les inconvénients liés aux très coûteuses infrastructures destinées à alimenter les locomotives et, notamment, d'importantes sous-stations destinées à transformer le triphasé du réseau général en continu basse tension ainsi qu'une caténaire lourdement armée apte à supporter des courants de forte intensité. C'est ainsi que l'électrification de Paris-Le Mans en 1938 fut pour Marcel Garreau l'occasion d'évoquer les autres systèmes développés en Europe - le $3 \mathrm{kV}$ continu des Italiens et l'alternatif haute tension à la fréquence 16 2/3 des Suisses et des Allemands - même si le système Parodi fut retenu in fine pour des raisons d'unification des types de locomotives ${ }^{10}$. En 1939, telle fut aussi la raison pour laquelle la SNCF avait décidé d'électrifier la ligne Paris-Lyon en utilisant le système Parodi. Cependant, les circonstances de la guerre ouvraient l'éventualité de remettre ce choix en question. Désormais, la SNCF fonctionnait sous l'étroit contrôle de l'occupant, en l'occurrence de la Wehrmacht Verkers Direktion, la direction des transports militaires allemands dont le chef à Paris (Albert Ganzenmüller) était un spécialiste de la traction électrique. C’est ainsi que les ingénieurs de la Reichsbahn proposèrent leur système d'électrification en alternatif monophasé à fréquence spéciale (16 2/3) pour la ligne Paris-Lyon et que la SNCF se voyait conviée à demander des devis de fabrication aux constructeurs de locomotives. Malgré tout, loin d'une fascination pour la technique allemande évoquée par certains historiens ${ }^{11}$, les ingénieurs français cherchaient les arguments qui leur permettraient d'éviter l'adoption du système germano-suisse sur leur réseau. Ainsi, dans ses Propos ferroviaires ${ }^{12}$, Louis Armand a raconté comment il cherchait à tergiverser en face de certains projets de l'occupant, parfois mégalomanes, comme celui d'un réseau à écartement de trois mètres électrifié en courant alternatif à fréquence industrielle $(50 \mathrm{~Hz})$ destiné à relier Paris et Berlin à l'Ukraine. De là, peut être, la proposition des ingénieurs de la SNCF de botter en touche en s'intéressant à une installation prototype en courant industriel réalisée en Allemagne avant la guerre sur la ligne du Höllental, mais qui malgré son

10- Marcel Garreau, «L'état actuel de l'électrification des chemins de fer», Revue générale des chemins de fer, fév. 1938.

11- Christian Bachelier, La SNCF sous l'Occupation allemande, 1940-1944, Rapport documentaire, Paris, IHTP-CNRS, 1996, 1534 p. ; 914 p. en 2 vol., + 2 vol. d'annexes. 12 Louis Armand, Propos ferroviaires, Paris, Fayard, 1969. 
intérêt économique n'avait pas donné satisfaction ${ }^{13}$. En effet, il faut rappeler que, depuis l'origine des électrifications ferroviaires au début du siècle dernier, les ingénieurs s'étaient heurtés au problème de la commutation des moteurs à collecteurs en courant monophasé qui induit une force contre-électromotrice d'autant plus néfaste que sa fréquence est élevée. Pour tourner la difficulté, le Dr ingénieur Hans BehnEschenburg de la firme suisse Oërlikon avait mis au point un moteur de traction apte à fonctionner à une fréquence spéciale de $162 / 3$ périodes par secondes selon des dispositions qui avaient servi à l'électrification des chemins de fer suisses puis allemands (en 1940, ces derniers disposaient de $1600 \mathrm{~km}$ de lignes électrifiées en 16 2/3, principalement en Bavière et en Silésie). Mais, entre temps, la Reichsbahn avait lancé des études pour utiliser le courant à fréquence industrielle 50 $\mathrm{Hz}$ sur les locomotives de la petite ligne du Höllental. C'est donc à visiter cette installation que le Dr Paul Müller, chef de la division traction de Siemens, conviait en 1943 un ingénieur de la firme SchneiderWestinghouse, Paul De Giacomoni, dûment mandaté par Louis Armand. Peu après, le 7 juin 1944 (date intéressante puisque le lendemain du D-Day en Normandie et une quinzaine avant l'arrestation d'Armand par la Gestapo) une réunion se tenait à Paris au siège de la SNCF pour décider : « d'installer le 50 pps. [périodes par seconde] sur des lignes de trafic moyen dont l'électrification en continu basse tension ne saurait se justifier, Clermont-Ferrand - Nîmes par exemple, pour lesquelles on pourra se contenter de locomotives moins puissantes qu'avec l'ancien système [le courant continu $1,5 \mathrm{kV}]^{14}$.»

Au lendemain de la guerre, lors du congrès pour l'avancement des sciences à Biarritz (1947), Louis Armand confirme que la SNCF a décidé de poursuivre ces essais - mais en équipant la ligne savoyarde d'Aix-les-Bains à la Roche-sur-Foron au lieu de celle des Cévennes ${ }^{15}$ car il précise, et c'est le point essentiel, qu'il s'agit d'un dispositif susceptible de représenter une économie de $50 \%$ en matière d'installations fixes par rapport au classique système Parodi ${ }^{16}$.

13- Une histoire parfaitement documentée par Yves Machefert-Tassin, Fernand Nouvion, Jean Woimant, Histoire de la traction électrique, tome I, Des origines à 1940, Paris, La Vie du Rail, 1980 ; t. 2, De 1940 à nos jours, ibid., 1986.

14- Note de la direction générale de la SNCF du 7 juin 1944 (Service O, n 4993), papiers A. Blanc.

15- La Savoie disposant d'un réseau de distribution haute tension (EDF) plus dense que la région cévenole, la ligne prototype de la SNCF bénéficierait de meilleures conditions d'alimentation. (Témoignage d'A. Blanc.)

16- Jean-François Picard, «Techniques universelles ou filières nationales ? Le cas de l'électrification des chemins de fer en Europe », Sciences \& $\sim$ Techniques en Perspective, I. I., 1997. 
Si le principal atout du monophasé $50 \mathrm{~Hz}$ est son économie, il reste à lever la principale hypothèque que représente le fonctionnement des locomotives. Sur le Höllental, les Allemands avaient essayé diverses solutions : groupes convertisseurs embarqués sur la locomotive (Krupp), transformation en courant continu grâce à des redresseurs (AEG, Brown Boveri), utilisation directe du $50 \mathrm{~Hz}$ dans les moteurs de traction (Siemens). Tandis que les locomotives à redresseurs provoquaient d'importantes perturbations dans le réseau d'alimentation électrique, la commutation de la machine à moteur direct ne donnait pas satisfaction. Comme, en cet immédiat après-guerre, de puissance occupée la France est devenue occupante, la SNCF décide de reprendre à son profit des essais de moteurs directs en Allemagne (Höllental) et en Autriche (Arlberg et Brenner). Ils sont confiés à la DETE.

«Il s'agissait de tester les moteurs directs pour le développement de la grande traction, se souvient Tessier. Nouvion voulait effectuer des démarrages lents en rampe $(2 \mathrm{~cm} / \mathrm{sec}$.) [...] On torturait les moteurs, les machines crachaient des étincelles grosses comme des petits pois, Nouvion s'amusait à taquiner les Allemands, il "occupait" la ligne. "Attends Coco ! J'ai pas terminé, on repart..." et c'est ainsi qu'il nous est arrivé de coller six heures de retard à l'Orient-Express qui attendait derrière ${ }^{17}$. »

La DETE disposait d'un train spécial composé de la voiture électrotechnique de la Reichsbahn et de la rame spéciale du général Koenig (ex-train spécial du Führer) avec ses facilités de logement et de restauration.

«On embarquait de nombreux invités, a raconté Nouvion, les ingénieurs de Schneider-Westinghouse, d'Alsthom, d'Oerlikon, de Siemens à laquelle s'agrégeaient des officiers supérieurs des armées occupantes ainsi que des "occupés" affamés. Comme on nourrissait bien nos gens, au fur et à mesure que nous circulions la foule croissait (et bien que l'alcool servi à la fin du repas provienne en droite ligne de l'I.G. Farben selon la déclaration du maitre d'hôtel) les convives se déclaraient satisfaits du cinquante périodes. Certains insistaient même pour être invités à la cinquante et unième ${ }^{18}$ !»

17- Témoignage de Marcel Tessier.

18- Fernand Nouvion, première « grande conférence » donnée à la SNCF (s.d.). Dossier Nouvion, AHICF. 
Au-delà de leur aspect folklorique, ces essais persuadent Nouvion que le moteur direct est la solution la plus simple pour faire de la traction en $50 \mathrm{~Hz}$ une conviction qu'il fait partager à Armand. Mais l'industrie ferroviaire française semble peu enthousiasmée par le nouveau système, de même que le constructeur suisse Brown Boveri, et la DETE va devoir se faire maittre d'œuvre. C'est le début d'une véritable aventure électrotechnique dont les ingénieurs de la SNCF seront les héros ${ }^{19}$. Pour la ligne de Savoie, elle met au concours la réalisation de deux machines à moteurs directs et d'une troisième à groupe convertisseur, cette dernière demandée par Marcel Garreau, soucieux de ménager une alternative en cas de difficultés avec les premières. Sage précaution, comme on le verra. Ces machines sont commandées en 1946 à la place de trois locomotives à vapeur conçues par André Chapelon et qui ne seront donc jamais construites (152 P et 232 à turbine). La firme suisse Oerlikon fournit à la SNCF une CC (6051) conçue par l'ingénieur Pierre Leyvraz, le Français Alsthom une CC (6052), enfin une locomotive à groupe convertisseur mono-continu est livrée par le Matériel de traction électrique (MTE) et Schneider-Westinghouse (SW), la BBB 6053.

Les essais de Savoie se sont révélés suffisamment probants pour inciter Louis Armand, devenu entre temps directeur général de la SNCF (1949), à lancer l'idée d'une électrification du réseau Nord-Est dans la perspective de la future Communauté européenne du charbon et de L'acier (CECA ${ }^{20}$. Lors d'un congrès électrotechnique organisé à Annecy à l'automne 1951, la SNCF propose d'électrifier en monophasé $50 \mathrm{~Hz}$ la ligne de Valenciennes à Thionville et à Coblence en Allemagne. Un accord en ce sens a été passé en février avec le secrétaire d'Etat aux Transports de la toute jeune République fédérale, le Dr Fröhne. Mais le congrès d'Annecy est l'occasion d'un débat entre exploitants français et allemands et aussi entre les « anciens » et les « modernes ». Hippolyte Parodi, le patriarche de l'électrification des chemins de fer français, devenu membre de l'Académie des sciences, a fustigé «la remise en cause, à l'issue d'une étrange discussion [?], d'un dispositif [le courant continu $1,5 \mathrm{kV}$ ] qu'on pensait définitivement adopté après la guerre de 1914-1918, contre $[s i c]$ le système allemand et suisse ». Fernand Nouvion est chargé de répondre à l'illustre académicien auquel on a soigneusement caché la difficile mise au point des locomotives à moteurs directs. Dans son intervention, le représentant de la DETE souligne que nos

19- L. Armand, Causerie aux X cheminots le 31 janv. 1952.

20- Le congrès d'Annecy suit d'un an la déclaration Schuman du 9 mai 1950 qui crée la Communauté européenne du charbon et de l'acier (CECA). 
locomotives à courant continu n'ont certes pas d'équivalent dans le monde, mais c'est pour mieux défendre l'alternatif « à la française » contre son rival «à l'allemande » :

«Certes la traction à $16^{2 / 3}$ est à la base du développement du $50 \mathrm{~Hz}$, dit Nouvion, mais le premier a ses faiblesses consécutives à l'emploi du moteur direct dont la force électromotrice de transformation n'a encore jamais été compensée à vitesse nulle et ne peut l'être complètement que pour des vitesses bien définies. Malgré des chiffres de puissance impressionnants qui peuvent faire illusion, les faiblesses du $16^{2 / 3}$ se manifestent en pratique par des charges remorquées peu importantes et cela dans des plages étroites de vitesse. On objectera : le $50 \mathrm{~Hz}$ ne risque t-il pas d'accroitre les faiblesses du $16^{2 / 3}$ ? Non, car au stade actuel des réalisations le moteur direct à $50 \mathrm{~Hz}$ se révèle supérieur aux meilleurs moteurs à $16^{2 / 3}$. D'autre part, il y a des avantages spécifiques aux machines à groupe et à moteurs asynchrones, un facteur de puissance élevé et une excellente utilisation de l'adhérence. Sur une machine à groupe les essieux sont en quelque sorte "embiellés" par la fréquence, comme dans les locos à vapeur... ${ }^{21}{ }^{2}$

Si l'exposé de Nouvion est bien reçu par les constructeurs germano-suisses, notamment Siemens et Oerlikon qui ont participé à l'avènement du nouveau système, il provoque la réticence des exploitants étrangers, que ce soit la Deutsche Bundesbahn (DB) ou les Chemins de fer fédéraux suisses (CFF). Le Dr Albert Peters, directeur du DBZentralamt de Munich, rappelle son peu d'enthousiasme pour les essais du Höllental ${ }^{22}$ : «Le $16^{2 / 3}$ a fait ses preuves et confirmé son utilité économique en Allemagne, souligne ce patron de la DB, et si les chemins de fer allemands, faisant preuve d'une grande largeur d'esprit, se sont déclarés prêts à étudier en relation avec la SNCF la ligne Diedenhofen [Thionville]-Trier-Koblenz, ils ne croient pas pouvoir en tirer qu'un bilan d'électrification en cinquante périodes se présente mieux qu'en 16 ${ }^{2 / 3}$. » Quant au Dr Meyer, le directeur des CFF, il confirme que son pays reste très satisfait du $16^{2 / 3}$, d'autant que son réseau est

21- Nouvion veut dire qu'une liaison électrique invisible remplace les bielles qui relient les essieux moteurs d'une locomotive à vapeur. L'ensemble des discussions du congrès d'Annecy est publié dans un numéro spécial de la Revne générale des chemins de fer, nov. 1951.

22- A. Peters était affecté à la WVD Paris sous l'occupation. Paradoxe de l'histoire, confronté à son hostilité pour le $50 \mathrm{~Hz}$, P. de Giacomoni qui avait été envoyé en Allemagne dix ans plus tôt dénonce «le manque d'esprit européen » de ses collègues d'outre-Rhin, cf. une copie de lettre de P. de Giacomoni à L. Armand (mai 1952) in Doss. Nouvion, AHICF. 
intégralement électrifié avec ce système. Tels sont les débuts d'une querelle d'ingénieurs d'où le chauvinisme de l'exploitant n'est jamais absent, mais où Nouvion va donner la mesure de ses talents de debater. Si le $16^{2 / 3}$ est considéré par les Allemands comme un système définitivement unifié, comment se fait-il qu'ils aient eu eux-mêmes l'idée d'essayer le $50 \mathrm{~Hz}$, demande t-il $?^{23}$ La discussion se poursuit dans la presse technique, le Dr ingénieur Förster défendant les vues de la DB dans la revue Elektrische Bahnen, celles de la SNCF étant développées par Nouvion dans la Revue générale des chemins de fer. Le débat atteint son point culminant lors des journées d'information organisées à Lille en 1955 pour célébrer l'électrification de la ligne Valenciennes-Thionville ${ }^{24}$. La DETE avait comparé les coûts de son électrification en $16^{2 / 3}$ et en $50 \mathrm{~Hz}$, étude d'où ressortait un bénéfice des deux tiers en coût d'équipement et de dix pour cent en frais d'exploitation au profit du nouveau système. Mais ces chiffres sont réfutés par les Allemands : «Le cinquante périodes réclamant des sectionnements de caténaire et des transformateurs spéciaux, n'offre que des solutions simplistes pour une électrification spartiate ", disent-ils, s'indignant de l'affirmation de Nouvion selon laquelle « des locomotives $50 \mathrm{~Hz}$ à quatre essieux seraient supérieures à des machines à six essieux en $16^{2 / 3}$. D'autant plus que les essais de différent types de locomotives par les Français laissent présager des difficultés pour unifier la technique ». Bref, les électriciens de la DB concluent : « qu'on ne saurait voir que tromperie [Betrug] dans le dossier de la $\mathrm{SNCF}^{25}$.»

En réalité, loin d'un prétendu bluff, la mise au point des locomotives de la ligne Valenciennes-Thionville est un succès justement revendiqué par la DETE et, s'il résulte de la comparaison entre divers types de locomotives (CC à groupes convertisseurs, BB à moteurs directs), il est surtout le triomphe d'une technologie appelée à révolutionner la traction en $50 \mathrm{~Hz}$, les redresseurs « ignitrons » des BB 12000. Évidemment, la solution du problème ne passait pas par le moteur direct comme l'espéraient Armand et Nouvion, mais par l'usage des redresseurs ainsi que l'avait imaginé Garreau. Les ignitrons sont des redresseurs scellés qui permettent de transformer le monophasé délivré par la caténaire en courant continu idéal pour alimenter les moteurs de locomotives.

23- Carnets techniques de Marcel Garreau, 8 avril 1952.

24- «L'électrification en courant monophasé $50 \mathrm{~Hz}$ de la ligne de Valenciennes à Thionville et les journées d'information de Lille (11-14 mai 1955)», Revue générale des chemins de fer, juillet 1955.

25- «Stellungnahm des Deutsche Bundesbahn zu den Worträgen auf des Informationstagung des Franszösischen Staatsbanhnen in Lille vom 11. bis 14 Mai $1955 »$, Elektrische Bahnen, mai 1956. 
En fait, c'est lors d'une mission d'étude chez Westinghouse aux ÉtatsUnis (1947) que Garreau avait eu l'idée d'acheter ces appareils afin d'en équiper une vieille automotrice testée sur la ligne de Savoie. Ainsi, on peut suivre Clive Lamming selon qui le cinquante périodes fut « un pari gagné in extremis grâce aux ignitrons $»^{26}$; sans eux le $50 \mathrm{~Hz}$ serait probablement resté un mode d'électrification à l'économie et non le système universel que nous connaissons aujourd'hui.

La réussite du monophasé à fréquence industrielle donne à la SNCF l'idée d'institutionnaliser sa collaboration avec les constructeurs de matériel électrique. Il s'agit d'ouvrir les marchés étrangers à la nouvelle technique. En avril 1954, sous l'impulsion de Marcel Garreau, la DETE suscite la constitution d'un « Groupement du $50 \mathrm{~Hz}$ » constitué avec AEG, Alsthom, Brown-Boveri, Jeumont, Schneider-Westinghouse, Siemens et les Ateliers de constructions électriques de Charleroi (ACEC). Fernand Nouvion y fera la preuve de ses capacités de vendeur. Dans le cadre du groupement, il participe à l'électrification de la banlieue d'Istamboul, à l'électrification des chemins de fer portugais (1955), il conçoit des locomotives destinées à l'URSS et à la Chine. En ExtrêmeOrient, il anime une mission technique chargée d'électrifier les Indian Railways. En 1957, la $1100^{\mathrm{e}}$ locomotive livrée en Inde par le Groupement $50 \mathrm{~Hz}$ sera solennellement baptisée Fernand Nouvion, un hommage dont il était très fier.

Le deuxième chapitre de l'histoire de la DETE concerne les aspects mécaniques de la traction électrique. Comme Louis Armand et Marcel Garreau, Nouvion estimait que le progrès du chemin de fer passait par l'accélération de la vitesse de ses trains. Mais si la traction électrique en monophasé haute tension permettait d'augmenter la puissance des locomotives, leur capacité à rouler vite restait liée à leur conception mécanique. C'est dans ce domaine qu'il s'est montré le plus innovateur, ce qui a fait dire à ses contemporains qu'il fut probablement «plus mécanicien qu'électricien ». Lui-même aimait répéter que : «le plus important dans une locomotive ce n'est pas le mode de traction, mais la partie mécanique dont l'électricité ne doit être que le serviteur ${ }^{27}$. » Avant-guerre, à l'époque de ses débuts, il existait deux types de locomotives électriques. Les unes étaient destinées à la traction des trains de marchandises, les autres à celle des rapides et express du trafic

26- Lamming, op. cit.

27- Fernand Nouvion, «Conférence donnée à l'École polytechnique en janvier 1956 (X Cheminots)», Doss. Nouvion, AHICF. 
voyageurs. Les premières étaient des machines à adhérence totale, à quatre ou six moteurs (BB, BBB, $\mathrm{CC}$ ) semi-suspendus dans les bogies, selon une disposition également propre aux automotrices (suspension par le nez). Très tôt Nouvion a travaillé sur les contraintes que ce genre de transmission faisait subir à la voie (chocs verticaux et latéraux) et à la durée de vie des moteurs (détérioration des bobinages). Lors de la mise au point des automotrices de ramassage il raconte qu'il lui arrivait de soulever les trappes de visite lors des marches d'essais.

«Pour me faire une opinion personnelle de ces suspensions par le nez, je me mettais debout sur un moteur en marche. C'était odieux, les vibrations intolérables ${ }^{28}$.»

Le second type était constitué de machines de vitesse, les fameuses 2D2 avec leurs quatre essieux moteurs, leurs moteurs entièrement suspendus (transmissions Büchli) et leurs bogies porteurs, selon une disposition inspirée des locomotives à vapeur. Mais ces reines de la vitesse étaient très lourdes, atteignant la limite théorique de charge par essieu sur le réseau français (22 t sur les 2D2 9100) et leur châssis rigide ne leur permettait guère de dépasser $140 \mathrm{~km} / \mathrm{h}$. D'où l'idée, surgie pendant la guerre, de marier le concept d'adhérence totale des machines à bogies, $\mathrm{BB}$ ou $\mathrm{CC}$, au principe de moteur entièrement suspendu des 2D2. C'est ainsi que, en vue de l'électrification Paris-Lyon, la DETE conçoit un type nouveau de locomotives à six essieux (CC) et à moteurs entièrement suspendus. Son prototype, la CC 7001 à transmission par biellettes Alsthom, sera livrée à la SNCF en 1948 pour donner la fameuse série des 7100. A l'instigation de Marcel Garreau ${ }^{29}$, la SNCF a aussi commandé à Schneider deux machines plus légères, des locomotives à quatre essieux (BB) équipés d'une transmission à anneaux dansants conçue par l'ingénieur André Jacquemin de la DETE, les 9003/4 en 1952. Celles-ci auront une descendance plus prolifique que les CC avec notamment la série 9200 dont il sera question plus loin.

La mise sous tension de la caténaire sur la ligne Paris-Lyon permet les premiers essais de vitesse puisque, avec la bénédiction d'Armand, Nouvion a obtenu de tester les nouvelles locomotives à des allures peu usuelles. En février 1954, le choix se porte sur la CC 7121 qui réalise une marche à $243 \mathrm{~km} / \mathrm{h}$ entre Dijon et Beaune. Au moment où la machine atteint sa vitesse maximum, Nouvion calcule que les balais du moteur voient défiler quatorze mille lames de collecteur par seconde, la limite d'emballement.

28- Fernand Nouvion, «Brouillon pour la grande vitesse » (s.d.), Doss. Nouvion, AHICF.

29- Carnets techniques de M. Garreau, juin-juillet 1949. 
«Mais, nous pouvions affirmer que jusqu'à des vitesses d'environ $270 \mathrm{~km} / \mathrm{h}$, il n'y avait pas de problèmes côté locomotives, sauf peut être avec les bandages des roues. »

Pourtant, il ne s'agit que d'un hors-d'œuvre ; l'année suivante voit la réalisation de l'extraordinaire « ruban bleu du rail», selon l'expression d'une revue de l'époque ${ }^{30}$. Les 28 et 29 mars 1955 une CC Alsthom (7107) et une BB Schneider (9004) sont mises en concurrence pour franchir la barre des $300 \mathrm{~km} / \mathrm{h}$. Nouvion a expliqué la raison de ces expériences :

«L'idée était d'abord de vérifier si, comme on le pensait à l'époque, les efforts sur la voie exercés par une locomotive variaient en fonction du carré de sa vitesse. Louis Armand, le directeur général de la $\mathrm{SNCF}$, voulait voir le maximum que l'on pouvait atteindre avec notre nouveau matériel. En 1954, entre Dijon et Beaune on avait déjà remarqué que le captage du courant posait des problèmes aux grandes vitesses, mais nous voulions aller encore plus loin et c'est la raison pour laquelle on a changé les rapports d'engrenage des moteurs ce qui nous libérait des problèmes de vitesse d'emballement. J'avais également remplacé les roues bandagées par des roues monoblocs, $j$ 'en avais marre de regarder si la marque que l'on faisait sur le bandage correspondait à celle du centre de la roue. Pour ces essais, on avait choisi la ligne des Landes (Bordeaux-Morcenx) dont les grands alignements permettaient d'aller très vite [...] Le premier jour, nous avons utilisé la CC, mais on a rencontré des difficultés de captage et on a fondu un pantographe. Je dois dire qu'on avait aussi commencé à percevoir une odeur de brûlé, indice de la destruction des silentblocs de la transmission et à l'arrivée à Morcenx, on pouvait nettement déceler le fluage des bielles de la transmission consécutif à la disparition du caoutchouc. En fait, la transmission Alsthom n'était pas homocinétique et bien que nous ayons supprimé le graissage des appuis latéraux, cela n’avait pas suffi à maintenir les bogies. Il y a d'ailleurs un chiffre qui n'a pu être divulgué à l'époque, celui de la vitesse vraiment réalisée par la CC, soit environ $270 \mathrm{~km} / \mathrm{h}$ au moment de la fusion du panto quand on a tout arrêté. Cette décision de confidentialité était justifiée par le fait que les conditions de marche avaient pâti des défaillances d'un appareil non fourni par le constructeur. Cela

30- Daniel Caire, «Le ruban bleu du rail. Deux locomotives électriques françaises atteignent $331 \mathrm{~km} / \mathrm{h}$ Lamothe-Morcenx, les 28 et 29 mars 1955 », Chemins de fer, revue de l'Association des amis des chemins de fer, $\mathrm{n}^{\circ} 191$ (mars-avril 1955). 
dit, il est heureux que je n'aie pas eu l'audace de remonter le panto avant pour continuer, la remise sous effort des bielles de transmission aurait pu entrainer leur rupture et des conséquences... imprévisibles ${ }^{31}$.»

L'essai de la BB Schneider réalisé le lendemain apporte aussi son lot d'émotions.

«Le lendemain on est reparti avec la BB et avec la même équipe de conduite que celle de la veille. C'était une erreur, mes types avaient fêté notre première marche et ils n'étaient pas très frais. En plus, ils n'étaient pas habitués à la commande à main de la BB. Résultat, à la suite d'une erreur de manipulation, on a démarré avec les moteurs shuntés et comme on a accéléré beaucoup plus lentement, lorsqu'on a enfin atteint les $300 \mathrm{~km} / \mathrm{h}$ le panto arrière était hors service. J'ai fait lever celui d'avant, lentement, j'étais inquiet à cause de ce qui était arrivé la veille. Mais ça a tenu et on a atteint $331 \mathrm{~km} / \mathrm{h}$ au moment du : "Coupez courant !" C'est d'ailleurs là que les accélérations subies par la loco ont brusquement changé de nature. Il s'est produit une sorte de mouvement de tamis qui a duré de manière pénible pendant une dizaine de secondes. On a cru avoir une avarie de transmission. Personnellement, j’ai craint le déraillement et, je le dis très franchement, j'ai eu peur. En fait, la transmission n'était pas responsable, mais la coupure brutale de l'effort de traction. C'est elle qui a produit cette déformation de la voie que l'on aperçoit sur certaines photos prises après les essais. Les appareils de mesure avaient enregistré des accélérations de $0,25 \mathrm{G}$ sur la machine et une poussée de six tonnes sur le rail, le tiers de la valeur de la charge sur l'essieu ${ }^{32}$ !»

Si ces expériences relèvent de l'exploit, elles s'avèrent aussi pleines d'enseignements.

" On apprend beaucoup lorsque on va sur le terrain au lieu de rester dans les bureaux, dit Nouvion. C'est l'essai de la BB qui nous a montré la nécessité de monter sur les bogies des amortisseurs anti-lacets à action rapide. Ce dispositif mis au point par Koni sera adopté ensuite sur tout le matériel SNCF, y compris le TGV. Les essais des Landes nous ont aussi appris que, pour aller

31- Fernand Nouvion, «Conférence donnée à l’École polytechnique en janvier 1956 (X Cheminots)».

32- Ibid. 
vite, il fallait des roues monoblocs, mais aussi qu'on devait surveiller le profil des surfaces de roulement. Ensuite qu'il y avait un problème de captage du courant, il fallait remplacer le continu basse tension par de l'alternatif haute tension [...] En 1990, le TGV a battu son record de $515 \mathrm{~km} / \mathrm{h}$ en prenant 700 Ampères à la caténaire, moi, j’en avais pompé plus de 5000 à $331 \mathrm{~km} / \mathrm{h}$ ! La conclusion était claire : il fallait passer au monophasé haute tension, ce que nous avons fait ${ }^{33}$.»

Cette recherche-développement menée par la DETE plutôt que chez les constructeurs apparait naturelle dans le monde cheminot, Nouvion dit qu' « on ne peut vivre le chemin de fer qu'en étant sans arrêt sur le terrain ", ce qui signifie que l'Exploitant est le seul à disposer d'un réseau lui permettant de faire des essais en vraie grandeur et, ajoutons, des crédits nécessaires au développement des nouvelles techniques. Il n'hésitait pas à fustiger l'abus des modèles mathématiques utilisés dans la conception d'un dispositif, comme le fait de réduire le paramètre " voie » dans l'étude de la dynamique d'un bogie à une simple constante, il rappelait à l'occasion qu'aucune voie ferrée ne ressemble à une autre ${ }^{34}$. Considérant que la conception du matériel doit relever d'abord des contraintes de l'exploitation et pas d'un quelconque cahier des charges remis aux constructeurs (comme c'est le cas à l'étranger), la DETE n’a jamais hésité à imposer ses conceptions à ses fournisseurs. Nouvion n'hésitait pas à régenter les bureaux d'études de l'Alsthom : «Écoutes, Coco, tu m'enquiquines, on fera comme ça et pas autrement... ${ }^{35}$. » A sa mise à la retraite en 1970, il anime Traction Export, le bureau d'études de l'entreprise Belfortaine devenue entre temps, et grâce à la SNCF, l'un des principaux constructeurs de locomotives dans le monde.

Auparavant, la DETE a dû convaincre la SNCF de tirer un profit commercial de ces essais de vitesse. Lorsque les Japonais mettent en service la ligne du Tokaïdo en 1964, Marcel Tessier constate que la grande vitesse ferroviaire devient une réalité quotidienne en Asie avant de l'être en Europe et il note que c'est la conséquence d'un curieux cheminement :

33- Témoignage F. Nouvion.

34- Fernand Nouvion, Conférence aux États-Unis en 1964, Doss. Nouvion, AHICF.

35- Témoignage de Raymond Garde. 
"Les ingénieurs japonais semblent avoir beaucoup appris de leurs visites en France. Ils étaient venus au congrès électrotechnique de Lille en 1955 et ils avaient appris qu'on pouvait aller vite tout en utilisant le $50 \mathrm{~Hz}$. On leur avait ouvert toutes les portes. On a eu pendant six mois un japonais à la DETE dont on s'est aperçu plus tard qu'il était chef d'études de traction électrique aux Japan National Railways ${ }^{36}$. »

Quant à Nouvion, il explique en toute modestie :

«lorsque les Japonais ont mis en service leur Tokaïdo, en fait, c'est comme s'ils m'avaient rendu un hommage silencieux! Les Japonais exploitaient des trains à plus de $200 \mathrm{~km} / \mathrm{h}$ alors que nous n'en avions pas un seul à 180 ! Ca faisait quand même drôle. En réalité la SNCF ne s'intéressait pas à la vitesse ! Je me suis donc adressé à Pierre Sudreau ${ }^{37}$ qui a convaincu le ministre des transports de l'époque, Edgar Pisani. Et c'est ce dernier qui a donné l'ordre à la SNCF de faire du $200 \mathrm{~km} / \mathrm{h}$ en service normal, alors que nous aurions pu le faire dix ans plus tôt... ${ }^{38}$.»

La fin des années soixante voit donc la mise en service commercial du Capitole, un train tracté à $200 \mathrm{~km} / \mathrm{h}$ par des BB 9200 à transmission Jacquemin (descendantes de la 9004 du record). Mais ce premier train à grande vitesse révèle les contraintes posées par la circulation sur un réseau conçu au siècle précédent.

"J'avais choisi la ligne la plus défavorable qui était Paris-Toulouse, explique Nouvion. On a pu faire du "200" entre Orléans et Vierzon, mais on traversait cette demière gare à $30 \mathrm{~km} / \mathrm{h}$ à l'heure. En fait, avec les $200 \mathrm{~km} / \mathrm{h}$ limités à la section Les Aubrais-Vierzon, on ne gagnait guère plus de quatre minutes sur l'ensemble du parcours, ce qui était ridicule. On a repris les essais, on a mesuré les efforts sur la voie, notamment dans la partie montagneuse et sinueuse ce qui nous a permis de gagner plus d'une demi-heure entre Limoges et Montauban ${ }^{39}$.»

36- Témoignage de Marcel Tessier. [Voir Terushi Hara, «Les échanges francojaponais de technologie ferroviaire dans l'après-guerre », in Dominique Barjot et Michèle Merger, avec la coll. de Marie-Noëlle Polino (sous la dir. de), Les Entreprises et leurs réseaux : hommes capitaux, techniques et pouvoirs, XIX'-XX' siècles, Mélanges en l'honneur de François Caron, Paris, Presses de l'université de Paris-Sorbonne, 1998, pp. 681-695 (N.d.l.R.).]

37- Ancien ministre du général de Gaulle, devenu président de la Fédération des industries ferroviaires en 1962.

38- F. Nouvion in Jean-François Picard et Alain Beltran, «D’où viens tu TGV ? Une histoire du TGV», RGCF, août-sept. 1994.

39- Ibid. 
L'augmentation de la vitesse des trains crée des contraintes en matière de signalisation (distances d'arrêt augmentées, visibilité des signaux, etc.) ce qui conduit la DETE à réaliser un cab-signal couplé à un système de pré-annonce. En revanche, comme le Capitole circule sur une voie de facture classique, dans une page significative rédigée plus tard Nouvion notera que ce qui étonne le plus les visiteurs étrangers en visite sur les lignes françaises à grande vitesse, c'est davantage cette voie ferrée banale que le matériel qui roule dessus. Quant au Capitole, il est suivi d'autres trains à grande vitesse au fur et à mesure de l'équipement des lignes et de l'amélioration du matériel roulant. Au début des années 1970, L'Aquitaine est lancé sur Paris-Bordeaux, un train auquel Nouvion a apporté deux innovations dont une majeure - les bogies monomoteurs des locomotives CC 6500 - l'autre sans suite - la pendulation des voitures " grand confort». En effet, les 6500 sont des locomotives aptes à rouler à $200 \mathrm{~km} / \mathrm{h}$ comme à hisser les trains de marchandise sur les rampes à $30 \%$ de ligne de la Maurienne, elles constituent un aboutissement du concept de «locomotive universelle » imaginé une vingtaine d'années plus tôt $t^{40}$.

L'idée de locomotive universelle a surgi des études menées à la DETE en vue de disposer de machines aptes à fonctionner sous les deux systèmes d'électrification (continu et alternatif $50 \mathrm{~Hz}$ ) comme à assurer tous types de services. L'objectif était de rationaliser le parc moteur de la SNCF en imaginant une machine électrique capable de tracter aussi bien des rapides internationaux de type Trans Europ Expres que des trains de banlieue ou des trains de marchandises. En fait l'idée de Nouvion était d'utiliser les ressources de la mécanique pour faire une locomotive universelle. Ultérieurement, les progrès de l'électrotechnique permettront d'envisager le recours à des solutions purement électriques pour obtenir le même résultat: voir les locomotives Sybic (36000) des années 1990. En 1958, les BB 16500 conçues pour l'électrification des lignes Paris-Strasbourg et Paris-Lille constituent la première série des locomotives universelles. Nouvion écrit à leur sujet :

«Cette locomotive n'est semblable à aucune de ses devancières, elle constitue aujourd'hui la forme la plus économique de locomotive universelle, grâce à un choix de nouveautés techniques qui en font un engin vraiment original. »

40- Fernand Nouvion, «La locomotive universelle », RGCF, juillet août 1959. 
Parmi ces innovations, l'usage de bogies monomoteurs qui, en simplifiant l'équipement électrique, permet de réduire les coûts de fabrication. Car il a imaginé d'équiper ses locomotives universelles d'un dispositif de changement du rapport de réduction qui permet de disposer de toute la puissance, soit à basse vitesse pour la traction marchandises, soit à grande vitesse pour le trafic voyageurs. De nombreuses séries de locomotives seront dotées de ce dispositif, parmi lesquelles on retiendra la série des CC 40100 (fournies également à la SNCB belge), des machines quadri-courant $(1,5 \mathrm{kV}$ courant continu, $3 \mathrm{kV}$ courant continu, $25 \mathrm{kV} 50 \mathrm{~Hz}, 15 \mathrm{kV} 16^{2 / 3}$ ) avec des bogies monomoteurs à deux rapports d'engrenage $(160 / 240 \mathrm{~km} / \mathrm{h})$, capables de circuler sur les différents réseaux électrifiés européens. Quant au bogie monomoteur, mais sans changement du rapport de réduction, il sera adopté sur une grande partie du matériel moteur de la SNCF, sur certaines automotrices de banlieue comme sur des locomotives diesel.

Bel objet mécanique, le bogie monomoteur requiert des normes de fabrication rigoureuses, surtout dans sa version à double rapport d'engrenages. Ainsi, la mise au point des BB 16500 a nécessité la participation active des ateliers de la SNCF, sans que cela prévienne les critiques répétées du service Matériel et Traction (MT) : leur tenue de voie vacillante leur a valu le sobriquet de « danseuses », tandis que leur dispositif de bi-réduction est victime de blocages chroniques. Lors d'une réunion interne organisée à Salins-les-Bains (Doubs) au début des années soixante, les participants se souviennent d'un Nouvion montant au créneau pour défendre «sa locomotive universelle »: «Personne au monde n'est capable de faire une machine aussi légère, aussi performante, aussi universelle que la 16500 et si vous avez des problèmes c'est que vous ne savez pas les entretenir », déclaration qui ne pouvait manquer de provoquer des remous dans l'assistance ${ }^{41}$. Jean Dupuy (X-Ponts) qui vient d'être nommé à la tête de la direction MT décide de résoudre le problème des bogies monomoteurs. Il est très critique vis-à-vis des conceptions techniques de Nouvion dont il conteste l'intérêt économique :

«Les bogies à deux rapports d'engrenage ne servent à rien si on relève la vitesse des trains de marchandise de $90 \mathrm{~km} / \mathrm{h}$ à 120 $\mathrm{km} / \mathrm{h}$ puisqu'on perd ainsi le bénéfice d'une économie de $8 \%$ sur le nombre de machines du $\operatorname{parc}^{42}$. »

41- Témoignage de Raymond Garde.

42- J. Dupuy à la réunion de la dir. MT-SNCF du 17 fév. 1964. Doss. Nouvion, AHICF. 
Le directeur du Matériel demande donc à la DETE de s'occuper désormais de locomotives conçues pour ne requérir que le minimum d'entretien et, au début des années 1970, le passage de la «locomotive universelle » à la nouvelle locomotive « sans entretien » sera symbolisé par les BB 15000 (en $50 \mathrm{~Hz}$ ) et par leur déclinaison en courant continu (7200) ou en bi-courant (22200). Dotée de bogies monomoteurs mais dépourvue du double rapport de réduction ${ }^{43}$, la série 15000 bénéficie d'autres améliorations techniques imaginées par la DETE ou par les services d'entretien qui en font le type de locomotive le plus réussi de la SNCF (il sera exporté sur des réseaux étrangers, hollandais ou marocain notamment). Signalons par exemple l'adoption de roulements à rouleaux sur les essieux moteurs et l'amélioration des moteurs de traction par l'usage de nouveaux isolants (vernis époxy et silicone pour isolation classe F, H), le soudage TIG des lames de collecteurs, etc. Toutes ces améliorations rendent les moteurs à la fois plus robustes, plus puissants et plus légers et feront dire à Nouvion qu'on peut « désormais piloter un moteur électrique en surveillant simplement la température de son collecteur $»^{44}$.

On peut se demander quelle fut la part de la Division d'études de la traction électrique dans la conception du train à grande vitesse, le célèbre TGV des années 1980. En réalité il semble que la DETE ne se soit qu'indirectement intéressée à sa genèse. Jean Bouley, le successeur de Dupuy à la direction du Matériel, le rappelait :

«Il est clair que ce concept de TGV ne cadrait pas avec les principes de la grande traction chers à la "bande de Nouvion". La DETE c'était les trains longs et lourds de l'avant-TGV. L'automotrice, la rame insécable ? C'était pour les pauvres, c'était la banlieue ${ }^{45}$ !»

Reste qu'à la fin des années 1960, Nouvion a participé à l'élaboration d'un TGV prototype, le fameux 001 à turbines à gaz, mais en renâclant. Hostile à la traction thermique, il rappelait qu'une machine électrique équivalait à trois ou quatre locomotives diesel en terme de rapport coût/performances et il n'eut de cesse de convaincre

43- Fernand Nouvion ; André Cossié ; Robert Dupont, «Les locomotives monophasées BB 15000 », RGCF, juin 1971.

44- Fernand Nouvion, "L'évolution des moteurs de traction à la SNCF », RGCF, déc. 1971.

45- Jean Bouley in Jean-François Picard et Alain Beltran, « D’où viens-tu T.G.V. ? Une histoire du TGV», RGCF, août-sept. 1994. 
la direction de la SNCF de réaliser un TGV «tout électrique » ${ }^{46}$. Cependant, poursuit Jean Bouley, «l'idée d'automotrice en configuration grande puissance faisait son chemin et Nouvion a vu ce qu'on pouvait tirer des dispositions de la rame articulée et notamment des bogies placés dans l'inter caisse », car cette disposition aurait permis d'envisager un TGV pendulaire. Dès la fin des années 1950, la DETE avait développé cette technique qui permet d'incliner les véhicules dans les courbes, ce qui améliore le confort des voyageurs, et on a signalé plus haut qu'elle avait prévu d'en équiper les voitures « grand confort » de L'Aquitaine. Mais le TGV ayant bénéficié de lignes nouvelles spécialement tracées pour la grande vitesse, le projet de rame pendulaire perdait de son intérêt. Autre exemple de suggestion non retenue, l'idée d'un TGV à bogies monomoteurs écartée par la direction du Matériel ${ }^{47}$. De même Nouvion critiquait-il le choix de moteurs synchrones auto-pilotés pour le TGV Atlantique :

« une impasse technologique, une sottise qui oblige les thyristors [les redresseurs de commande] à travailler contre-nature [à transformer du monophasé en triphasé]. D’ailleurs "leur" électronique n'est pas infaillible, "leurs trucs" en "trons" et en "stors" sont tellement compliqués qu'ils deviennent un facteur majeur de pannes ${ }^{48}$.»

Derrière l'ironie grinçante de l'ingénieur perçait probablement l'amertume de ne pas avoir terminé sa carrière au grade de directeur à la SNCF.

Il reste qu'au moment de conclure cette biographie on ne saurait minorer le rôle exceptionnel de Fernand Nouvion dans la modernisation du chemins de fer. Du fameux record de 1955 aux performances quotidiennes des TGV d'aujourd'hui, il a directement participé aux progrès techniques qui expliquent la renaissance d'un vénérable moyen de transport inventé un siècle plus tôt, mais rajeuni grâce à la traction électrique et aux très grandes vitesses. Même si son égotisme lui fit

46- Fernand Nouvion, « Rames expérimentales à turbines à gaz », RGCF, janvier 1970 et, du même, «Electric or diesel traction the right basis for comparison », Railway Gazette International, oct. 1971.

47- Voir par exemple un projet d'article de Nouvion pour la RGCF d'avril 1984 revu et corrigé par J. Dupuy et R. Garde, Doss. Nouvion, AHICF.

48- Témoignage de F. Nouvion [Le Musée des Arts et Métiers (Paris) donne à entendre aux visiteurs, à la sortie du département « transports », un témoignage de F. Nouvion sur les grandes vitesse, enregistré en 1990 (N.d.l.R.).]. 
souvent revendiquer les innovations des autres dans la Division d'études de la traction électrique, son dynamisme de manager fut l'ingrédient indispensable pour moderniser l'infrastructure technique dans la grande entreprise publique chargée du transport sur rail. En ce sens on peut dire que sans la DETE, mais aussi sans le sens de la publicité d'un Nouvion, il n'y aurait vraisemblablement pas eu de TGV. De même, c'est la prééminence technique de la SNCF qui a permis à l'industrie ferroviaire française d'acquérir la stature internationale qu'on lui connait aujourd'hui et, si la mode en est peut-être passée en ces temps de libéralisme triomphant et de privatisations tous azimuts, on devait rappeler le rôle de ses ingénieurs dans l'histoire récente. 